\title{
Algas arribadas da Praia do Pacheco, Ceará
}

\section{Arribadas algae from Pacheco beach, Ceará, Brazil}

\author{
Gabriela de Sousa Ferreira ${ }^{(1)}$; Paulo Ovídio Batista de Brito ${ }^{(2}$; Francisco Ícaro Carvalho \\ Aderaldo (D); Pedro Bastos de Macedo Carneiro ${ }^{(D)}{ }^{3}$; Adriana Marques Rocha ${ }^{4}{ }^{4}$; Franklin Aragão \\ Gondim (1D 4
}

${ }^{1}$ Graduandos em Engenharia Ambiental e Sanitária do Instituto Federal de Educação, Ciência e Tecnologia do Ceará, Maracanaú; gabrieladesousaf@ hotmail.com; icaroaderaldo16@ hotmail.com. ${ }^{2}$ Mestrando em Energias Renováveis do Instituto Federal de Educação, Ciência e Tecnologia do Ceará; paulobatistaengenharia@gmail.com. ${ }^{3}$ Professor na Universidade Federal do Piaú; pedrocarneiro@ufpi.edu.br. ${ }^{4}$ Professor no Instituto Federal de Educação, Ciência e Tecnologia do Ceará, Maracanaú; adrianamr2@yahoo.com.br; aragaofg@yahoo.com.br.

\section{A R T I G O}

Recebido: 18/03/2019

Aprovado: 18/02/2020

Palavras-chave:

Macroalgas

Adubo orgânico

Nutrição vegetal

Key words:

Macroalgae

Organic fertilizer

Plant nutrition

\section{R E S U M O}

As macroalgas são seres importantes em seus habitats, pois possuem grande relevância ecológica, uma vez que, nos ecossistemas aquáticos, são fonte de nutrientes e os principais organismos fotossintetizantes. A Praia do Pacheco em Caucaia, município da região metropolitana de Fortaleza-CE, compõe a planície litorânea do nordeste brasileiro. Nesta região, é comum que as macroalgas que se desprenderam de seu substrato se acumulem durante a maré baixa, denominadas de algas arribadas. Esse trabalho objetivou identificar a comunidade de algas arribadas da Praia do Pacheco, avaliando seu potencial nutricional para a formulação de fertilizante para plantas. Nessa perspectiva, foram realizadas duas coletas manuais durante a maré baixa. Uma em abril de 2017 e a outra em outubro de 2018. As amostras foram identificadas e, posteriormente, três das espécies de maior incidência foram analisadas em termos de macro e micronutrientes. Foram encontrados dezoito táxons, sendo: quatorze algas vermelhas, duas algas verdes e duas algas pardas. As espécies encontradas em maior quantidade foram a Hypnea pseudomusciformis, Gracilaria cearensis e Ulva fasciata. Quando analisadas, observou-se que possuem altos níveis de nutrientes com potencial para fins agrícolas. A região estudada apresentou-se como um ambiente rico em macroalgas e em biomassa que devem ser utilizada de forma consciente para garantir sua sustentabilidade.

A B S T R A C T
Macroalgae are important beings in their habitats, because they have great ecological relevance.
In the aquatic ecosystems, they are source of nutrients and the main photosynthetic organisms.
The Pacheco beach in Caucaia, a municipality in the metropolitan region of Fortaleza-CE,
composes the coastal plain of the Brazilian Northeast. In this region, it is common for the
macroalgae that have detached from their substrate to accumulate during low tide, called algae
arribadas. This aim of this work was to identify the algae arribadas community of Praia do
Pacheco by evaluating its nutritional potential for the formulation of fertilizer for plants. In this
perspective, two manual collections were carried out during the low tide. One in April 2017 and
the other in October 2018 . The samples were identified and, subsequently, three of the species of
higher incidence were analyzed in terms of macronutrients and micronutrients. It was obtained
eighteen taxa, being: fourteen red algae, two green algae and two brown algae. The species found
in greater quantity were Hypnea pseudomusciformis, Gracilaria cearensis and Ulva fasciata.
When analyzed, it was found that there are high nutrients with potential for agricultural purposes.
The studied region presented a rich environment in macroalgae and in biomass that must be used
in a conscious way to guarantee its sustainability.

\section{INTRODUÇÃOO}

As algas são seres importantes em seus habitats, uma vez que, nos ecossistemas aquáticos, são fonte de nutrientes e os principais organismos fotossintetizantes. Possuem representantes unicelulares e pluricelulares, sendo destaque os grupos de macroalgas: Feofícea ou algas pardas; Rodófitas ou algas vermelhas e Clorófitas ou algas verdes (MOTA et al., 2014).

As algas marinhas estão inseridas em muitos ramos da sociedade, sendo utilizadas para fins diversos como: alimentação; cosméticos; fármacos; biotecnologia e agricultura (DAPPER et al., 2014). Como fertilizantes, as macroalgas atuam na nutrição vegetal como reguladoras,

\section{Revista Verde}

ISSN 1981-8203

Pombal, Paraíba, Brasil v. 15 , n.2, abr.-jun., p.208-214, 2020

doi: $10.18378 /$ rvads.v15i2.6472 
proporcionando uma resposta mais efetiva a condições de estresse. Dentre as alterações benéficas destaca-se o desenvolvimento do sistema radicular (GALINDO et al., 2019).

Na faixa litorânea, durante a maré baixa, é comum que as macroalgas que se desprenderam de seu substrato e se acumulem, denominadas de algas arribadas (BRITO et al., 2018). As algas apresentam estruturas denominadas apressórios que atuam na fixação dos organismos ao solo. No entanto, ao perderem essas estruturas, as algas ficam sujeitas à grande variação de umidade, temperatura, salinidade, luz e às forças abrasivas do movimento marítimo da região, formando massas flutuantes e visíveis em padrões estriados ou em camadas no costão rochoso (RAVEN et al., 2014).

A Praia do Pacheco compõe a planície litorânea do nordeste brasileiro, está localizada no município de Caucaia, no Ceará, e integra a Região Metropolitana de Fortaleza. Entre a linha de praia e o mar, paralelamente, encontram-se os arenitos de praia cuja principal função natural é a proteção contra a erosão marinha. Tais estruturas são definidas como um material rochoso alongado e estreito, formado por areias de praia cimentadas por carbonatos. Nestas áreas ocorre o abrasamento, acumulando montantes de algas arribadas diariamente (GOES; FERREIRA, 2017).

O conhecimento das estruturas de comunidades de macroalgas é essencial para o desenvolvimento de monitoramento e manejo ambiental. Há a necessidade de mapear a incidência de cada espécie para que estratégias de recuperação e conservação sejam efetivas, entretanto estudos focados nesse eixo ao longo da costa cearense são escassos, em especial na Praia do Pacheco. Assim esse trabalho objetivou identificar a comunidade de algas arribadas da Praia do Pacheco, bem como avaliar seu potencial nutricional para formulação de fertilizante para plantas.

\section{MATERIAL E MÉTODOS}

O estudo foi realizado na Praia do Pacheco-CE (Figura 1), em uma área de $6324 \mathrm{~m}^{2}$, onde se realizaram as coletas de material, os registros fotográficos da região, as medições da faixa de areia e da amplitude da maré. A faixa de areia que se estende por $50 \mathrm{~m}$ e a faixa rochosa, aparente durante a maré seca, de $170 \mathrm{~m}$ de arenitos de praia onde ocorre o arribamento das algas.

Figura 1. Mapa de localização do ponto de coleta na Praia do Pacheco, Ceará, Brasil.

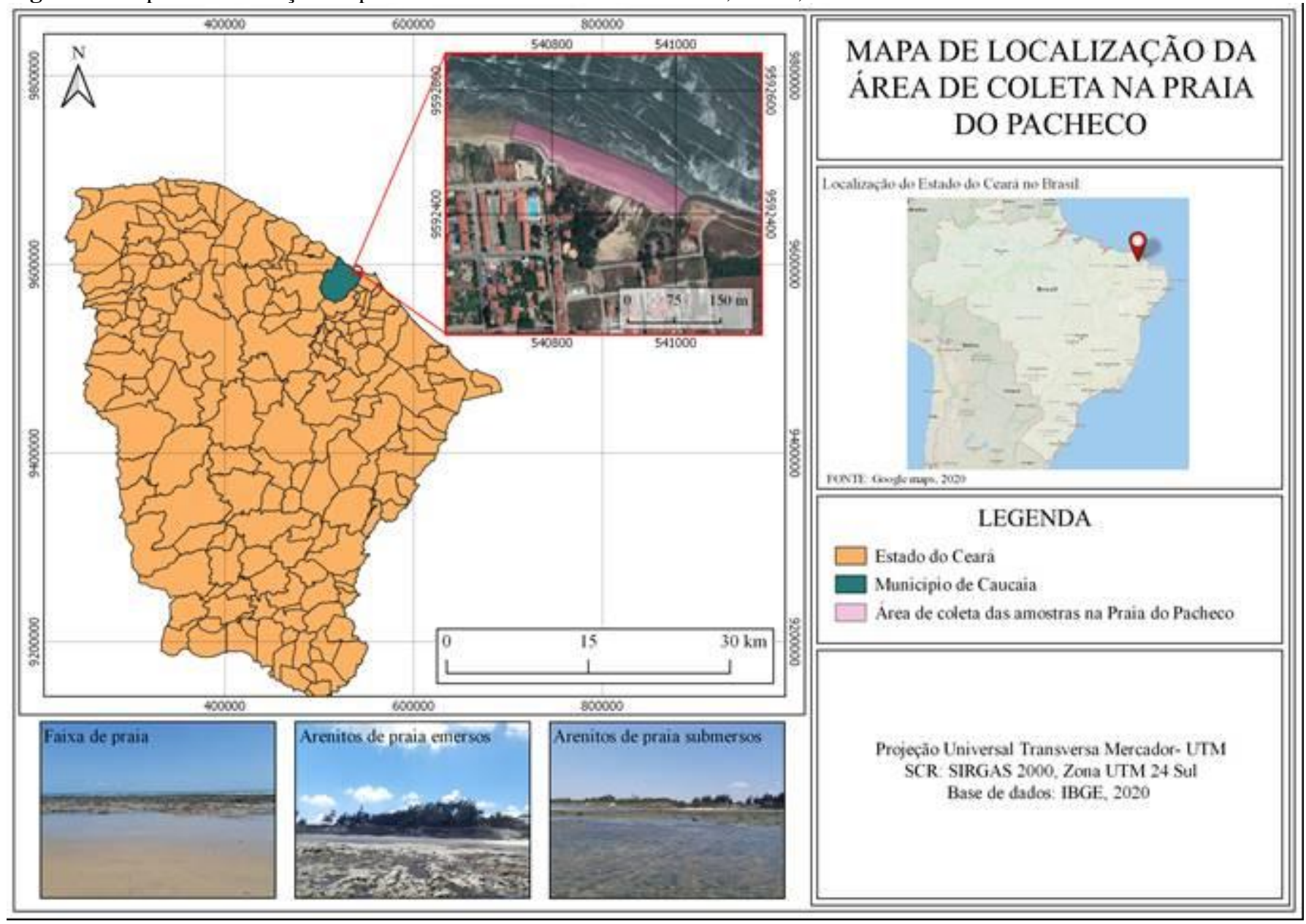

Fonte: Autores, 2019.

As coletas das amostras combinadas de algas arribadas foram feitas de forma manual e aleatória, durante a maré baixa. Foram realizadas 2 coletas. A primeira no dia 15 de abril de 2017, entre 13:00 e 15:00h com maré a 0,4 m e lua minguante (BRASIL, 2017) e a segunda no dia 08 de outubro de 2018, entre as 10:00 e 12:00h com maré $0,0 \mathrm{~m}$ e lua minguante (BRASIL, 2018). Os valores de maré foram obtidos na tábua de marés do Porto do Pecém-CE.

As amostras coletadas foram levadas ainda in natura, preservadas a $4^{\circ} \mathrm{C}$ em caixa térmica, ao herbário do Instituto de Ciências do Mar (Labomar) da Universidade Federal do Ceará (UFC) para identificação de espécies. 
Durante o período de visitas ao local, observou-se visualmente que três espécies predominaram. Estas foram separadas e levadas ao laboratório de Bioquímica e Fisiologia Vegetal do Instituto Federal de Educação, Ciência e Tecnologia do Ceará (IFCE) campus Maracanaú. Para a realização das análises de micro e macro nutrientes foram produzidas amostras combinadas. Retiraram-se pequenas quantidades aleatórias de diferentes regiões da área experimental totalizando, aproximadamente $25 \mathrm{~L}$ de cada espécie.

As amostras de cada espécie foram lavadas em água corrente, posteriormente secas em estufa de circulação forçada à $80^{\circ} \mathrm{C}$ até que se atingissem massa constante. $\mathrm{O}$ material foi triturado e, juntamente com um fertilizante comercial, encaminhado ao Laboratório de Solo da UFC para a análise de macro (Nitrogênio, sódio, potássio, cálcio e magnésio) e micronutrientes (Ferro, cobre, zinco e manganês), ambas realizadas conforme a metodologia de Malavolta et al. (1997). Os cálculos das concentrações de nutrientes foram realizados através de curvas padrão com concentrações conhecidas dos elementos analisados.

\section{RESULTADOS E DISCUSSÃO}

Os recifes costeiros, como os arenitos de praia, são constituídos a partir da cimentação de areia de praia por carbonato de cálcio $\left(\mathrm{CaCO}_{3}\right)$ ou óxido de ferro. Estudos realizados com a alga marinha Halimeda opuntia em um recife de arenito do sudoeste do Oceano Atlântico, verificaram resultados positivos usando a alga como produtora de $\mathrm{CaCO}_{3}$ (CARNEIRO et al., 2018). Sabe-se da existência de um consórcio com as macroalgas marinhas que ajudaram na construção e sedimentação dos recifes. É um ecossistema com fortes interações ecológicas (VASCONCELOS et al., 2011). No município de Icapuí-CE, pesquisas definiram algumas das principais interações e serviços prestados pelos bancos de algas, sendo eles: controle de erosão, retenção marinha, refúgio de vida silvestre, ciclagem de nutrientes, dissipador de matéria e energia, controle biológico, recursos genéticos, segurança alimentar e é base para o modo de vida de algumas comunidades litorâneas (LIMA et al., 2014).

$\mathrm{Na}$ figura 2A é possível observar a parte da faixa de areia e os arenitos de praia. Agregado a essas rochas havia siris, ouriços, peixes de pequeno porte e até espécies pequenas de polvos. Juntamente com esses animais, uma parte das macroalgas ainda permanecia fixa ao substrato (Figura 2B) e com a diminuição da maré, ao longo do tempo, as algas se desprendiam e ficavam expostas ao sol (Figura 2C), facilitando a decomposição.

Segundo dados dos boletins de balneabilidade disponibilizado pela SEMACE (2018), as praias do litoral oeste do estado do Ceará, em sua maioria, estão próprias para banho. Sendo definida próprias, pois ao analisar um conjunto de amostras de um mesmo local, por cinco semanas, $80 \%$ houve no máximo 1.000 coliformes termotolerantes por 100 $\mathrm{mL}$ da amostra. Contudo, durante esse estudo, a Praia do Pacheco foi visitada tanto na estação chuvosa quanto seca, respectivamente, abril e setembro. Percebeu-se que, no mês de abril, as algas arribadas possuíam uma grande quantidade de resíduos (Figura 2D) quando comparadas as algas em setembro (Figura 2E). Essa diferenciação é oriunda da ampliada vazão dos rios no período chuvoso, sabe-se da proximidade do ponto de coleta do Rio Ceará, que por sua vez carreiam maior quantidade de resíduos descartados nos centros urbanos.

Figura 2. Arenitos de praia visíveis na maré seca. (A) Ulva fasciata e Hypnea pseudomusciformis fixadas ao substrato. (B) Algas arribadas, principalmente a espécie Ulva fasciata secando ao Sol. (C) Algas arribadas durante o período de Abril/17. (D) Algas arribadas durante o período de Setembro/18. (E) Ponto de lixo no início da faixa de areia. (F)

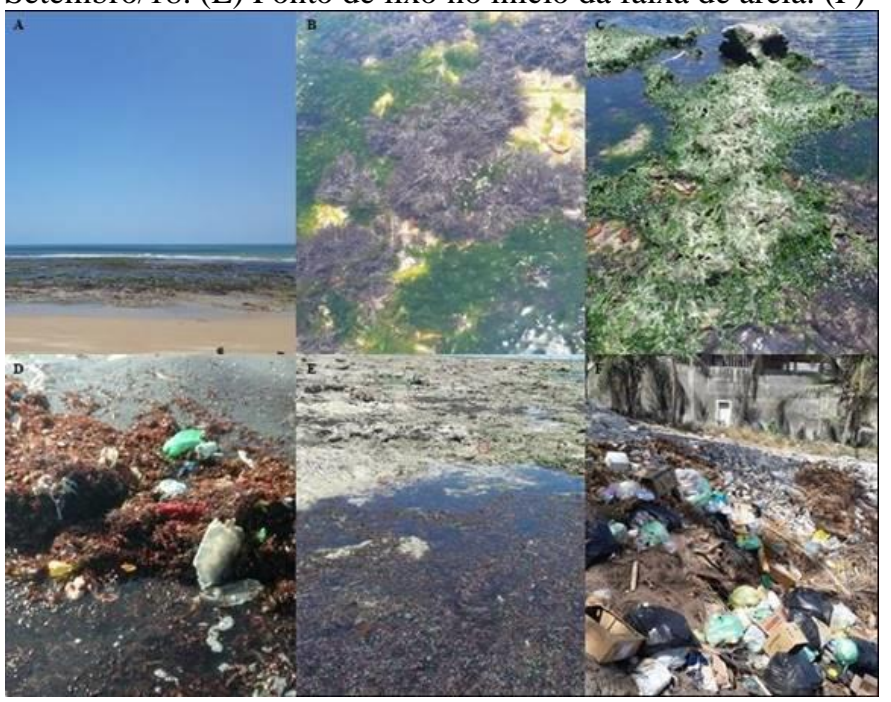

Fonte: Autores, 2019.

Embora quase não houvesse lixo na água no período de setembro, foi encontrada no início da faixa de praia (Figura 2F), uma grande quantidade de resíduos a céu aberto, enquadrados nas tipologias desde resíduo domiciliar a de construção civil. Para Silva et al. (2012), que avaliaram o impacto antrópico em algas marinhas, a diversidade de espécies se torna comprometida com a intensidade do impacto antrópico, pois, em áreas onde as perturbações são intensas, apenas sobreviverão as algas mais bem adaptadas.

Foram identificados dezoito táxons distintos de algas arribadas na Praia do Pacheco, sendo 6 do gênero Gracilaria. Portugal et al. (2016), estudaram e detectaram como um impacto negativo as variadas pressões antropogênicas, representadas pelo índice de pressão ambiental relativa, sobre a comunidade bentônica ao longo da costa tropical do Atlântico Sul. Contudo, segundo Miloslavich et al. (2011), a média brasileira é de 10,6 espécies a cada $100 \mathrm{Km}$ de costa, demonstrando assim que a região estudada tem uma elevada riqueza em macroalgas marinhas.

As algas identificadas foram em maior quantidade do filo das Rodófitas, sendo: Pterocladiella beachiae (Figura 3A), Tricleocarpa sp. (Figura 3B), Cryptonemia crenulata (Figura 3C), Corallina pannizoi (Figura 3D), Hypnea pseudomusciformis (Figura 3E), Halymenia sp (Figura 3F), Botryocladia occidentalis (Figura 3G), Gracilaria domingensis (Figura 3H), Gracilaria caudata (Figura 3I), Gracilaria cearenses (Figura 3J), Gracilaria cerviconis (Figura 3K), Gracilaria curtissiae (Figura 3L), Gracilaria suzannae (Figura 3M), Gelidiopsis gracilis (Figura 3N). Do filo Clorófita foram encontradas duas espécies, sendo elas Ulva faciata (Figura 3O) e Caulerpa prolifera (Figura 3P). E do filo das Feófitas Spatoglossum schroederi (Figura 3Q) e Lobophora variegata (Figura 3R). 
Figura 3. Táxons identificados: Pterocladiella beachiae. (A) Tricleocarpa sp. (B) Cryptonemia crenulata. (C) Corallina pannizoi. (D) Hypnea pseudomusciformis. (E) Halymenia sp. (F) Botryocladia occidentalis. (G) Gracilaria domingensis. (H) Gracilaria caudata. (I) Gracilaria cearenses. (J) Gracilaria cerviconis. (K) Gracilaria curtissiae. (L) Gracilaria suzannae. (M) Gelidiopsis gracilis. (N) Ulva faciata. (O) Caulerpa prolifera. (P) Spatoglossum schroederi. (Q) Lobophora variegata. (R)

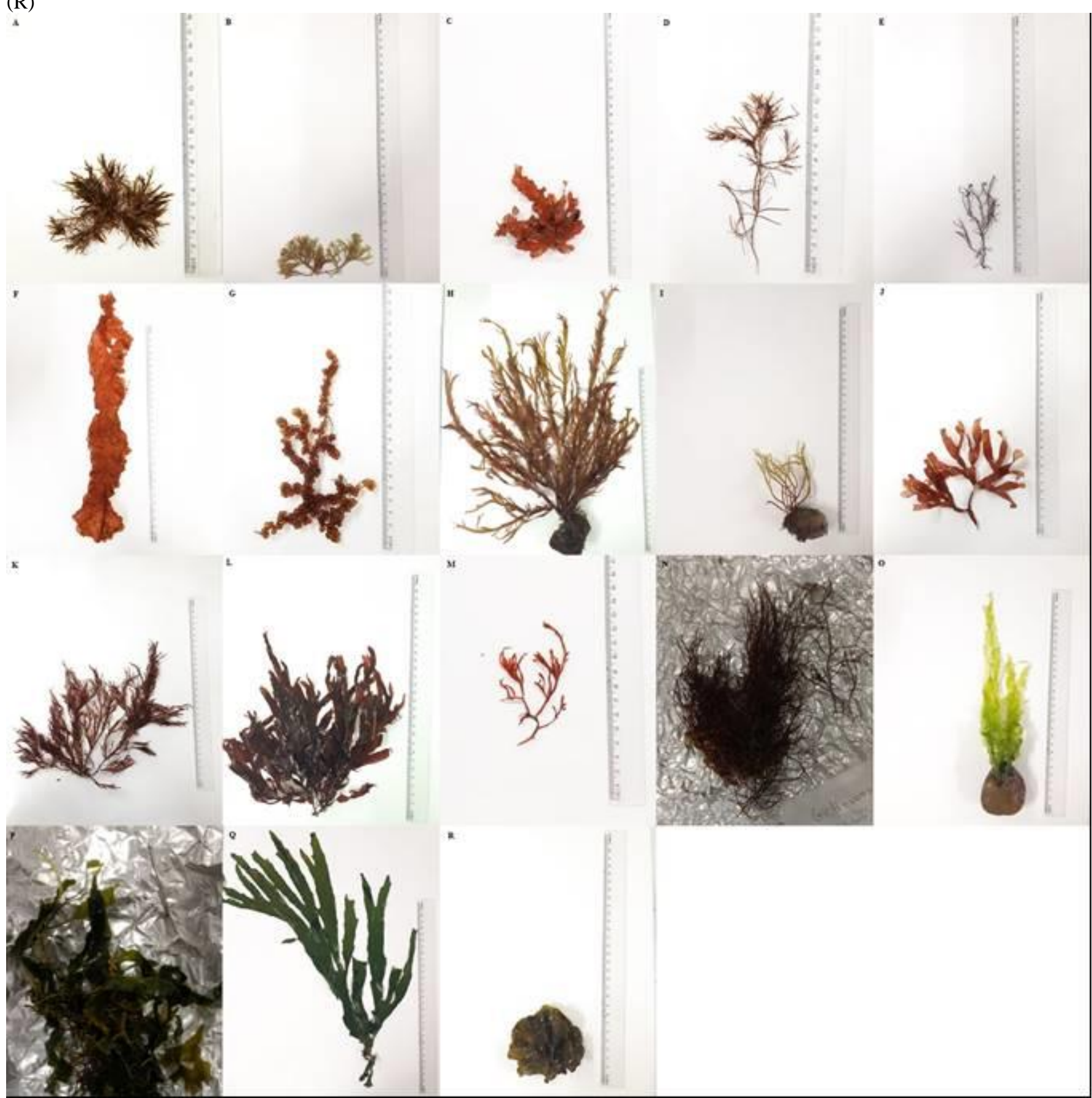

Fonte: Autores, 2019.

Entre as espécies identificadas, a divisão que mais se destacou foi a Rodófita. As algas desse filo contém ficoeritrina como pigmento que é responsável por seu tom avermelhado. Apresentam a clorofila do tipo "a", "d" e carotenóides. São primordialmente macroscópicas, marinhas e bentônicas (TEXEIRA, 2012). Possuem concentrações elevadas de proteína em comparação com outras algas podendo assim ser utilizada como fonte de alimento para animais (VASCONCELOS; GONÇALVES, 2013).
Três das algas encontradas estão presentes em maior proporção, são elas: H. pseudomusciformis (Figura 3E), G. cearensis (Figura 3J) e U. fasciata (Figura 3O).

A espécie encontrada visualmente em maior volume foi a H. pseudomusciformis (Figura 3E). Tem ocorrência desde o litoral do Rio Grande do Sul até o litoral do Maranhão, em áreas de infra e mesolitoral sobre rochas ou como epífitas em outras espécies de algas (JESUS et al., 2014). Em ambientes com boa conservação, as algas corticadas são representadas por este gênero e, por suas paredes celulares conterem carragenana do tipo kappa, possuem grande potencial 
industrial (COSTA et al, 2012). A carragenana possui propriedades gelificantes sendo utilizada na indústria farmacêutica, de cosmético e alimentícia (SANTOS et al., 2018).

A G. cearensis (Figura 3J) é uma espécie comum em recifes rasos e ao longo do nordeste da costa brasileira que possui potencial para a produção de ágar e atividade antibiótica (SOARES et al., 2015). Algas desse gênero têm alto rendimento e taxas de crescimento rápidas (XU et al., 2009).

A $U$. fasciata (Figura 30) é uma macroalga marinha de incidência no mesolitoral, conhecida como alface do mar (MARTINS et al., 2013). É um gênero considerado oportunista e tolerante a grandes variações ambientais. Em regiões eutrofizadas, a Ulva tende a ser a dominante (MARMITT et al., 2015).

De acordo com Nova et al. (2014), as macroalgas encontradas na praia, podem ser aproveitadas para o processo de compostagem e como complemento para a adubação em diversas atividades agrícolas, como no cultivo de plantas de Moringa oleifera. Brito et al. (2018), avaliaram de forma positiva a utilização de resíduo orgânico de algas arribadas para cultivo de girassol (Helianthus annus) quando comparadas a um fertilizantes comercial.

Com intuito de verificar a viabilidade das espécies encontradas em maior incidência para a utilização na produção de fertilizantes orgânicos, analisaram-se as concentrações de macro e micronutrientes (Tabela 1). Verificaram-se concentrações elevadas de nutrientes. As macroalgas em comparação com as plantas terrestres apresentam um alto rendimento, pois vão possuir baixa exigência para a produção de tecidos suportes e possuem capacidade de absorver nutrientes ao longo de toda a sua área superficial (BORINES et al., 2011).

Tabela 1. Macro e micronutrientes das espécies G. cearensis, H. pseudomusciformis, U. fasciata e de um fertilizante comercial.

\begin{tabular}{|c|c|c|c|c|c|c|c|c|c|}
\hline & \multicolumn{5}{|c|}{ g. $\mathrm{Kg}^{-1}$} & \multicolumn{4}{|c|}{ mg. Kg ${ }^{-1}$} \\
\hline & $\mathrm{N}$ & $\mathrm{P}$ & $\mathrm{K}$ & $\mathrm{Ca}$ & $\mathrm{Mg}$ & $\mathrm{Fe}$ & $\mathrm{Cu}$ & $\mathrm{Zn}$ & $\mathrm{Mn}$ \\
\hline Gracilaria cearensis & 51,2 & 2,14 & 18,00 & 12,95 & 7,68 & 383,7 & 1,3 & 50,1 & 571,7 \\
\hline Hypnea pseudomusciformis & 45,6 & 1,88 & 14,00 & 6,22 & 5,55 & 391,9 & 18,9 & 41,3 & 436,1 \\
\hline Ulva fasciata & 45,1 & 1,38 & 10,60 & 6,13 & 15,10 & 267,3 & 29,9 & 1,8 & 385,5 \\
\hline Fertilizante comercial & 2,2 & 8,43 & 8,10 & 1,52 & 1,85 & $3.455,5$ & 1,80 & 89,3 & 380,1 \\
\hline
\end{tabular}

As concentrações de nitrogênio $(\mathrm{N})$, fósforo $(\mathrm{P})$, potássio (K) tiveram seus valores mais elevados na $G$. cearensis. Ao analisar a nutrição vegetal, constata-se que o nitrogênio é um dos elementos exigidos em elevadas concentrações no solo pelas plantas. $\mathrm{O} \mathrm{N}$ é essencial na constituição dos aminoácidos, nucleotídeos e clorofila, além de participar do processo enzimático. O fósforo é um dos principais componentes do trifosfato de adenosina (ATP) e é um nutriente necessário nos processos de armazenamento, transferência de energia e proteção da membrana das células das plantas. O potássio contribui para um maior crescimento vegetal, atuando na área foliar, nos teores de clorofila e na retenção de água (VIECELLI, 2017).

Os valores de cálcio $(\mathrm{Ca})$ foram superiores nas duas algas vermelhas. É um nutriente indispensável para a manutenção da estrutura vegetal promovendo o fortalecimento, principalmente das raízes e das folhas, além do auxílio no equilíbrio ácido e básico entre o meio e a planta (DIAS et al., 2012). Já o magnésio ( $\mathrm{Mg}$ ), com maiores valores presentes na $U$. fasciata, atua na pigmentação, faz parte da clorofila e sua deficiência traz à cultura um aspecto amarelado (SERRAT et al., 2002). Tanto o Ca quanto o $\mathrm{Mg}$ são nutrientes necessários na calagem do solo.

Os micronutrientes Ferro (Fe), Cobre $(\mathrm{Cu})$, Zinco $(\mathrm{Zn})$ e Manganês (Mn) funcionam como íons no solo, cuja função é de ativar o transporte de elétrons. Estudos feitos por Bugs et al. (2018) avaliaram de forma positiva a utilização de algas como biossorvente na remoção de metais pesados.

$\mathrm{O} F$ possui função estrutural, participação na fotossíntese e na ativação enzimática. $\mathrm{O} \mathrm{Zn}$ atua no crescimento e frutificação. $\mathrm{O} \mathrm{Cu}$ e o $\mathrm{Mn}$ aumentam da resistência a pragas e participam da fotossíntese (SERRAT et al., 2002).

Formulados de NPK tem comumente recomendações de valores de 15, 2, $10 \mathrm{~g} . \mathrm{Kg}^{-1}$ respectivamente para o nitrogênio, fósforo e potássio. Os valores obtidos para a G. cearenses foram maiores para os três nutrientes, sendo 3,4 vezes mais elevados para o nitrogênio, 1,07 vezes para o sódio e 1,8 vezes para o potássio. As demais espécies de algas foram superiores tanto no nitrogênio quanto no potássio, o que demonstra o potencial dessa biomassa como fertilizante orgânico. As elevadas concentrações de nitrogênio presentes nas algas garantem que pequenas concentrações supram a exigência nutricional.

Quando as algas foram comparadas ao fertilizante comercial (Tabela 1), verificaram-se valores mais elevados para a maioria dos macronutrientes nas algas. Para o nitrogênio, verificou-se que as algas foram superiores ao fertilizante. A $G$. cearensis apresentou 23,3 vezes mais nitrogênio que o fertilizante. Já para os micronutrientes, de modo geral, não foram detectadas grandes diferenças entre as concentrações presentes nas algas e no fertilizante. Contudo, para o ferro, os valores foram bem mais elevados no fertilizante. Estudos feitos por Jucoski et al. (2016), comprovaram a toxicidade do ferro em elevadas concentrações no cultivo de plantas de Eugenia uniflora, sendo caracterizada pelo bronzeamento foliar e escurecimento das raízes. Krohling et al. (2016), também comprovou efeitos negativos do excesso de ferro em raízes, caules e folhas no cultivo de Coffea canephora. Laurett et al. (2017), comprovou que valores acima de 5090,4 mg. $\mathrm{kg}^{-1}$ de ferro no cultivo de alface Vitória de Santo Antão e da rúcula Rococó reduziram o desenvolvimento do cultivo.

\section{CONCLUSÕES}

A Praia do Pacheco tem elevada riqueza de macroalgas marinhas, apesar da presença de pontos de lixo. Foram detectados dezoito táxons, sendo quatorze algas vermelhas, duas algas verdes e duas algas pardas. As espécies 
encontradas em maior quantidade são a $H$. pseudomusciformis, G. cearensis e U. fasciata.

As análises químicas indicam o potencial nutricional para produção de fertilizantes orgânicos a partir da biomassa das espécies $G$. cearensis, $H$. pseudomusciformis e $U$. fasciata.

A utilização desse material para produção de fertilizante orgânico mostrou-se viável. Contudo, é uma matéria prima que deve ser utilizada de forma consciente para garantir sua sustentabilidade, bem como, dos ecossistemas que dependem dela.

\section{AGRADECIMENTO(S)}

Ao Instituto de Ciências do Mar (Labomar) da Universidade Federal do Ceará (UFC) pelo apoio na identificação das macroalgas.

\section{REFERÊNCIAS}

BICUDO, C. E. de M.; MENEZES, M. Catálogo de plantas e fungos do Brasil. Instituto de Pesquisa Jardim Botânico do Rio de Janeiro, v.1, p. 49-60, 2010.

BORINES, M. G.; LEON, R. L. de; MCHENRY, M. P. Bioethanol production from farming non-food macroalgae in Pacific island nations: Chemical constituents, bioethanol yields, and prospective species in the Philippines. Renewable and Sustainable Energy Reviews, vol. 15, n. 9, p. 4432-4435, 2011. 10.1016/j.rser.2011.07.109.

BRASIL. Centro de Hidrografia da Marinha. Disponível em: $<$ https://www.marinha.mil.br/chm/dados-do-

segnav/dados-de-mare-mapa>. Acesso em: 08 de setembro de 2018.

BRITO, P. O. B. D.; MARTINS, K.; BARBOSA, R. M.; ARRUDA, J. F. D.; CARNEIRO, P. B. D. M.; GONDIM, F. A. Growth, relative chlorophyll content and concentration of inorganic solutes in sunflowers plants supplemented with marine macroalgae organic residue. Ceres, v. 65, n. 5, p. 395401, 2018. 10.1590/0034-737x201865050003.

BUGS, L. C.; CUPERITINI, P. M.; WOLF, T. C.; TREICHEL, H. Uso da biomassa de algas como biossorvente para remoção de metais pesados: uma revisão. CIATEC-UPF, v. 10, n. 1, p. 53-67, 2018. 10.5335/ciatec.v10i1.7183.

COSTA, I. O.; CAIRES, T. A.; FILHO, G. H. P.; NUNES, J. M. C. Macroalgas bentônicas associadas a bancos de Hypnea musciformis (Wulfen) JV Lamour.(Rhodophyta-Gigartinales) em duas praias do litoral baiano. Acta Botanica Brasilica, v. 26 , n. 2, p. 493-507, 2012. 10.1590/S010233062012000200025.

CARNEIRO, P. B. de M.; PEREIRA, J. U.; MATTHEWSCASCON, H. Standing stock variations, growth and $\mathrm{CaCO}_{3}$ production by the calcareous green alga Halimeda opuntia. Journal of the Marine Biological Association of the United Kingdom, v. 98, n. 2, p. 401-409, 2018. $10.1017 / \mathrm{S} 0025315416001247$.
DIAS, J. S.; NEVES, I.; SILVEIRA, V. H. da. Nutrientes Do que as plantas precisam. Unifertil, n.2, p.1-10. 2012.

DAPPER, T. B.; PUJARRA, S.; OLIVEIRA, A. J. de; OLIVEIRA, F. G. de; PAULERT, R. Potencialidades das macroalgas marinhas na agricultura: revisão. Agronegócios e Meio Ambiente, v.7, n.2, p. 295-313, 2014.

JESUS, P. B. de; MACHADO, G. E. M. M.; MUNIZ, A. de R. Macroalgas marinhas como indicadores de impactos ambientais em Itacoatiara, Niterói, RJ: subsídios para futuros programas de monitoramento ambiental. Caderno de Estudos Geoambientais, v.4, n.1, p.67-80, 2013.

JESUS, P. B. de; SCHNADELBACH, A. S.; NUNES, J. M. de C. O gênero Hypnea (Cystocloniaceae, Rhodophyta) no litoral do estado da Bahia, Brasil. Sitientibus série Ciências Biológicas, v.13, p.1 - 21, 2014. DOI: http://dx.doi.org/10.13102/scb215.

JUCOSKI, de O.; CAMBRAIA, J.; RIBEIRO, C.; OLIVEIRA, J. A. de. Excesso de ferro sobre o crescimento e a composição mineral em Eugenia uniflora L. Ciência Agronômica, v. 47, n. 4, p. 720-728, 2016. 10.5935/1806$\underline{6690.20160086}$.

GALINDO, F. S.; FILHO, M. T.; CARVALHO, M.; BUZETTI, S.; ALVES, C. J.; GARCIA, C. M. DE P.; NOGUEIRA, L. M. Extrato de algas como bioestimulante na nutrição e produtividade do trigo irrigado na região de Cerrado. Colloquium Agrariae, v.15, n.1, p.130-140, 2019. 10.5747/ca.2019.v15.n1.a277.

GOES $^{1}$, E. R.; JUNIOR, A. V. F. Caracterização morfossedimentar da plataforma continental Brasileira. Revista Brasileira de Geografia Física, v.10, n.5, p. 1595$1613,2017$.

KROHLING, C. A.; EUTRÓPIO, F. J.; FIGUEIRA, F. F.; CAMPOSTRINI, E.; DOBBSS, L. B.; RAMOS, A. C. Níveis tóxicos de ferro em lavouras de café conilon (Coffea canephora L.) em solos de tabuleiros costeiros. Coffee Science, v. 11, n. 2, p. 255-266, 2016. 10.25186/cs.v11i2.1088.

LIMA, A. P. da S.; CARNEIRO, R. N.; MEIRELES, A. J. de A. Ecossistema banco de algas e identidade territorial no município de Icapuí/Ce: Comunidade Tradicional pesqueira e meio técnico-científico-informacional. Eletrônica do PRODEMA, v.8, n.2, p. 2, p. 35-49, 2014.

LAURETT, L.; FERNANDES, A. A.; SCHMILDT, E. R.; ALAMEIDA, C. P.; PINTO, M. L. P. B. Desempenho da alface e da rúcula em diferentes concentrações de ferro na solução nutritiva. Ciências Agrárias/Amazonian Journal of Agricultural and Environmental Sciences, v. 60, n. 1, p. 4552, 2017. 10.4322/rca.2466

MALAVOLTA, E.; VITTI, Godofredo César; OLIVEIRA, Sebastião Alberto de. Avaliação do estado nutricional das plantas: princípios e aplicações. [S.1: s.n.], 1997. 
MILOSLAVICH, P.; KLEIN, E.; DÍAZ, J. M.; HERNANDEZ, C. E.; BIGATTI, G.; CAMPOS, L.; CARRANZA, A. Marine biodiversity in the Atlantic and Pacific coasts of South America: knowledge and gaps. PloS one, v.6, n.1, p. e14631, 2011. 10.1371/journal.pone.0014631.t001.

MARTINS, G. M.; PATARRA, R. F.; ÁlVARO, N. V.; PRESTES, A. C.; NETO, A. I. Effects of coastal orientation and depth on the distribution of subtidal benthic assemblages. Marine Ecology, v. 34, n. 3, p. 289-297, 2013. $\underline{10.1111 / \text { maec. } 12014 .}$

MOTA, N. S.; FIGUEIREDO, T. V. B.; MACHADO, B. A. S.; DRUZIAN, J. I. Macroalgas marinhas comestíveis: tendências tecnológicas. Cadernos de Prospecção, v. 7, n. 2, p. 118, 2014. 10.9771/S.CPROSP.2014.007.013.

MARMITT, D. J.; FREITAS, E. M.; MARCZINSKI, F.; FLESH, A.; BLASI, É. A. R. Avaliação quali-quantitativa de algas marinhas macrófitas ocorrentes na Praia da Vigia, Garopaba/SC. CEPSUL-Biodiversidade e Conservação Marinha, v. 4, n. 1, p. 5-15, 2015.

NOVA, L. L. M. V.; COSTA, M. M. da S.; COSTA, J. G. da; AMORIM, E. C. da S.; GUEDES, É. A. C. Utilização de "Algas Arribadas" como alternativa para adubação orgânica em cultivo de moringa (Moringa oleifera Lam.). Ouricuri, v. 4, n. 3, p. 68-81, 2014.

PORTUGAL, A. B., CARVAlHO, F. L., CARNEIRO, P. B. de M., ROSSI, S., Soares, M. de O. Increased anthropogenic pressure decreases species richness in tropical intertidal reefs. Marine environmental research, v. 120, p.44-54, 2016. 10.1016/j.marenvres.2016.07.005.

RAVEN, P. H.; EVERT, R. F.; EICHHORN, S. E. Biologia Vegetal. 8. ed. Rio de Janeiro: Guanabara Koogan, 2014. cap. 15 , p. $628-712$.

SERRAT, B. M.; LIMA, M. R. D.; GARCIAS, C. E.; FANTIN , E. R.; CARNIERI, I. M. R. S. A.; PINTO, L. S. Conhecendo o solo. 1ed. Curitiba : UFPR/Setor de Ciências Agrárias/Departamento de Solos e Engenharia Agrícola, 2002. p. 27.
SILVA, I. B.; FUJII, M. T.; MARINHO-SORIANO, E. Influence of tourist activity on the diversity of seaweed from reefs in Maracajaú, Atlantic Ocean, Northeast Brazil. Farmacognosia, v. 22, n. 4, p. 889-893, 2012. 10.1590/S0102$\underline{695 X 2012005000078}$.

SOARES, L. P.; GURGEL, C. F. D.; FUJII, M. T. Taxonomic reassessment of Gracilaria cearensis (Rhodophyta, Gracilariales), a poorly defined yet common flattened species based on morphological and molecular analysis including topotype collections. Phytotaxa, v. 201, n. 4, p. 241-255, 2015. 10.11646/phytotaxa.201.4.1.

SANTOS, F. P.; BRUNIERA, L. B.; GARCIA, C. E. R. Carragena: uma visão ambiental. Terra \& Cultura: Cadernos de Ensino e Pesquisa, v. 24, n. 47, p. 58-68, 2018.

SEMACE. Índice de praias próprias e impróprias para banho na Região Metropolitana de Fortaleza. Disponível em: $<$ https://www.semace.ce.gov.br/wp-

content/uploads/sites/46/2018/10/Boletim_RMF.pdf>. Acesso em: 02 de setembro de 2018.

TEIXEIRA, V. L. Produtos naturais de algas marinhas bentônicas. Virtual de Química, v. 5, n. 3, p. 343-362, 2012. 10.5935/1984-6835.20130033.

VASCONCELOS, E. R. T. P. P.; REIS, T. D. V.; GUIMARÃES-BARROS, N. C., SOARES, L. P.; MIRANDA, G. E. C.; COCENTINO, A. L. M. Métodos de amostragem para comunidades de macroalgas marinhas em recifes de arenito. Engenharia de Pesca, v.6, n.1, p. 17-29, 2011. 10.18817/repesca.v6i1.383.

VASCONCELOS, B. M. de F.; GONÇALVES, A. A. Macroalgas e seus usos: alternativas para as indústrias brasileiras. Revista Verde, v. 8, n. 5, p. 125 - 140, 2013.

VIECELLI, C. A. Guia de deficiências nutricionais em plantas. 1ed. Paraná: Assoeste, 2017. p. 111.

XU, Y.; WEI, W.; FANG, J. Effects of salinity, light and temperature on growth rates of two species of Gracilaria (Rhodophyta). Chinese Journal of Oceanology and Limnology, v. 27, n. 2, p. 350 - 355, 2009. 10.1007/s00343009-9116-0. 